\title{
La progresión de las concepciones de los futuros profesores de primaria sobre la metodología de enseñanza
}

\author{
Ana Rivero ${ }^{1}$ \\ arivero@us.es \\ Pilar Azcárate ${ }^{2}$ \\ pilar.azcarate@uca.es \\ Rafael Porlán ${ }^{3}$ \\ rporlan@us.es \\ Rosa Martín Del Pozo ${ }^{4}$ \\ rmartin@edu.ucm.es \\ Joao B. Harres ${ }^{5}$ \\ joao.harres@pucrs.br \\ Emilio Solís Ramírez ${ }^{6}$ \\ esolis@us.es
}

\begin{abstract}
Resumen
Este artículo describe y analiza la evolución de las concepciones de profesores de Primaria en formación acerca de la metodología de enseñanza. En concreto, aunque se han estudiado tres categorías (concepto de actividad, organización de
\end{abstract}

1 Professora del Departamento de Didáctica de las Ciencias de la Universidad de Sevilla.

2 Professora del Departamento de Educación de la Universidad de Cádiz.

3 Professor del Departamento de Didáctica de las Ciencias de la Universidad de Sevilla.

4 Professora del Departamento de Didáctica de las Ciencias de la Universidad Complutense de Madrid.

5 Professor de la Universidad de Porto Alegre.

6 Professor del Departamento de Didáctica de las Ciencias de la Universidad de Sevilla. 
las actividades y concepto de recurso didáctico), se presentan los resultados obtenidos en relación a una de ellas: la organización de las actividades. El estudio se ha realizado con cinco equipos de futuros profesores que participaron en cursos de formación de orientación constructivista e investigadora en varias universidades. En los resultados obtenidos se detectan muy diversos itinerarios en los procesos de cambio y la presencia de dos fuertes obstáculos, que la enseñanza es la causa directa del aprendizaje, y el absolutismo epistemológico. El estudio nos permite señalar algunas implicaciones para la formación inicial de los profesores.

Palabras-Clave: formación inicial de profesores; conocimiento profesional; estrategias metodológicas; secuenciación de actividades.

\section{Introducción}

Son numerosos los estudios que han descrito y analizado las concepciones de los profesores sobre la ciencia y sobre su enseñanza y aprendizaje. Estas concepciones se caracterizan por ser estables y difíciles de cambiar pues, en la mayoría de los casos, proceden de su experiencia interiorizada como alumnos y no de la formación recibida para ser docente (AGUIRRE; HAGGERTY, 1995; GUSTAFSON; ROWEL, 1995; HASWEH, 1996; MELLADO, 1996; YERRICK; PARKE; NUGENT, 1997; JORAM; GABRIEL, 1998; PORLÁN; RIVERO, 1998; SZYDLIK; SZYDLIK; BENSON, 2003; BESWICK, 2006). Así mismo, es un criterio bastante aceptado que la formación de profesores ha de organizarse en torno a dichas concepciones para facilitar su evolución (LEMBERGER et al., 1999; DUIT; TREAGUST, 2003; AMBROSE, 2004). En relación con esto, nuestro equipo de investigación se ha venido ocupando desde hace tiempo de tres ámbitos de estudio relacionados y complementarios:

a) el estudio de las concepciones didácticas y epistemológicas de los profesores. En este ámbito hemos identificado una tendencia mayoritaria, basada en una visión empiro-positivista de la ciencia y en un modelo transmisivo de enseñanza, y otras minoritarias, con una vision más relativista de la ciencia y más centradas en el papel activo del alumno 
(PORLÁN et al., 1998; Martín del Pozo; Porlán, 2001; Porlán; Martín del Pozo, 2002; 2004; 2006);

b) la caracterización del conocimiento del profesorado como un conocimiento práctico profesional (CPP). Este conocimiento se organiza en torno a los Problemas Prácticos Profesionales (PPP), y se construye por superación gradual de obstáculos epistemológicos y didácticos (PORLÁN et al., 1996; 1998; PORLÁN; RIVERO; MARTÍN DEL POZO, 1997; AZCÁRATE, 1999a; HARRES et al., 2005);

c) el diseño y experimentación de estrategias de formación que promuevan la construcción del CPP. Estas estrategias se fundamentan en un modelo de formación que denominamos "Formación de Profesores para Investigar la Práctica" (FOPIP) (PORLÁN et al., 1996; PORLÁN; RIVERO, 1998; PIZZATO; HARRES, 2007), basado en dos ideas claves: - organizar el proceso formativo en torno a "Problemas Prácticos Profesionales" (PPP) (PETERSON; TREAGUST, 1998; WATTERS; GINNS, 2000). Los que consideramos más relevantes para la construcción del conocimiento profesional son aquellos relacionados directamente con aspectos vinculados al currículo escolar: las ideas de los alumnos, los contenidos de enseñanza, la metodología de enseñanza, la evaluación, el diseño de unidades didácticas, la planificación de un curso completo y la definición del modelo didáctico personal (PORLÁN; GARCÍA, 1992; PORLÁN; RIVERO, 1998; MARTÍN DEL POZO; RIVERO, 2001; PORLÁN; MARTÍN DEL POZO, 2006),

- facilitar la construcción de respuestas a los PPP planteados gracias al contraste con informaciones tanto teóricas como prácticas y mediante procesos de negociación del conocimiento, en ambientes democráticos y críticos (WATTS; JOFILI, 1998).

Con este marco de fondo, nos hemos centrado en los últimos años en el estudio de la progresión de los futuros profesores cuando participan en procesos formativos coherentes con el modelo FOPIP. Es lo que Niedderer, Goldberg y Duit (1992) denominan estudios del aprendizaje como consecuencia de intervenciones específicas. Para ello, hemos diseñado un curso denominado "Concepciones de los Alumnos e Innovación Curricular" (CAIC) que concreta y adapta dicho modelo a los 
contextos formativos en los que trabajamos. En el CAIC se abordan los siguientes "Problemas Prácticos Profesionales": las ideas de los alumnos, los contenidos escolares y la metodología de enseñanza.

En este artículo vamos presentar y analizar los resultados obtenidos en relación con uno de ellos: la metodología de enseñanza.

\section{La metodología de enseñanza}

En la actualidad, existe un consenso bastante generalizado entre los investigadores acerca de algunos de los principios de lo que podríamos denominar metodología deseable. Nos referimos a los siguientes (WATTS; JOFILI, 1998; DUIT; TREAGUST, 2003):

a) poner el foco en el que aprende; es decir, pasar de una enseñanza centrada en el profesor a una enseñanza centrada en el alumno (sus ideas, afectos, intereses, necesidades, etc.);

b) generar un ambiente de aprendizaje que favorezca las interacciones (entre profesores y alumnos, entre los propios alumnos, entre distintos tipos de saberes, etc.);

c) promover la construcción de conocimientos mediante procesos de negociación democrática, lo que implica un cierto relativismo epistemológico y el cuestionamiento de las relaciones de poder habituales.

En esta línea, consideramos que resultan especialmente adecuadas las propuestas metodológicas basadas en la investigación de problemas abiertos, cercanos, interesantes y relevantes para los alumnos. Para el tratamiento de estos problemas en el aula es necesario negociar los significados que aportan mejores soluciones, fomentando así la actividad y el protagonismo real de los alumnos en los procesos de enseñanzaaprendizaje. En este enfoque, las actividades constituyen el elemento organizador de la vida del aula, la unidad de programación. Mediante ellas se definen las interacciones didácticas, los contenidos a trabajar, las actuaciones concretas, la distribución del tiempo y del espacio, los recursos necesarios, etc. Cañal (2000, p. 5) define las actividades como "procesos de flujo y tratamiento de la información (orientados, interactivos y organizados) característicos del sistema aula". 
Desde otras visiones, sin embargo, las actividades se conciben de manera distinta. Por ejemplo, desde enfoques transmisivos se consideran como situaciones protagonizadas por los alumnos para comprobar y/o aplicar la información que transmite el profesor (AZCÁRATE, 1999b). Para muchos profesores, las actividades son sólo situaciones especiales, ligadas a la práctica (hands-on activities), que se caracterizan por ser interesantes y motivadoras para los alumnos (APPLETON, 2002). Pero, desde perspectivas constructivistas, como acabamos de señalar, la actividad es la unidad del proceso metodológico y su sentido es facilitar a los alumnos la construcción del conocimiento.

Para conseguir esto, las actividades deben relacionarse entre sí de manera coherente, configurando un conjunto articulado. Aunque es habitual que los profesores organicen la secuencia temporal en el aula en función de la lógica de los contenidos, desde el enfoque de enseñanza que venimos defendiendo, el hilo conductor de la secuencia de actividades lo constituye la evolución de las ideas de los alumnos. Los contenidos se consideran entonces como herramientas para abordar los problemas investigados y para promover el desarrollo de las competencias necesarias.

En el desarrollo de cada actividad es necesario utilizar diversos recursos didácticos. La validez de los recursos depende de la finalidad perseguida y no sólo del recurso en sí mismo. Consideramos, pues, que los recursos que se pueden usar en la enseñanza son muy variados (instrumentos, objetos, documentos, lugares, personas, acciones, etc.), y que es muy importante seleccionar aquellos que resulten más adecuados, en cada caso, para facilitar la construcción de conocimientos.

Por todo lo que acabamos de exponer, consideramos que para caracterizar las distintas opciones metodológicas son relevantes las siguientes categorias: definición y sentido de la actividad, criterio de organización de las actividades (el hilo conductor de la secuencia) y definición y sentido de los recursos didácticos, y son las que abordamos en nuestro estudio. En esta comunicación nos vamos a centrar en describir y analizar las progresiones de futuros profesores con relación a una de estas categorías (organización de las actividades) cuando participan en el curso CAIC.

Según los estudios revisados los futuros profesores inician su 
formación con visiones sobre la enseñanza coherentes con un enfoque de caracter transmisivo (PETERSON; TREAGUST, 1998; MEYER et al., 1999; HAEFNER; ZEMBAL-SAUL, 2004). En menos ocasiones lo hacen con enfoques inductivos, coherentes con la idea de descubrimiento, o incluso se pueden detectar tendencias próximas al constructivismo (GUSTAFSON; ROWEL, 1995; SKAMP; MULLER, 2001a; PORLÁN; RIVERO; MARTÍN DEL POZO, 1998). Debemos señalar también que frecuentemente suelen aparecer contradicciones entre lo que los futuros profesores de primaria declaran acerca de sus enfoques o modelos educativos y lo que diseñan para sus aulas (HEWSON; HEWSON, 1987) y entre lo que diseñan y lo que hacen (MELLADO, 1996; BRYAN; ABELL, 1999). Las declaraciones pueden estar alejadas de los modelos transmisivos - llegando a ser próximas a perspectivas constructivistas, pero las prácticas no lo consiguen (HANEY; MCARTHUR, 2002; SO; WATKINS, 2005; CHENG et al., 2009). Estas contradicciones pueden ser debidas a la ausencia de referentes prácticos de carácter innovador que apoyen y orienten las intenciones de cambio, por lo que los futuros profesores acaban enseñando como han sido enseñados y son pocos permeables en la práctica a las ideas innovadoras. Así, el estudio de Skamp y Muller (2001 b) resalta que los factores que los estudiantes de profesor señalan como más relevantes en sus concepciones acerca de un buen profesor de ciencias, son sus experiencias previas como alumnos y sus experiencias durante las prácticas de enseñanza en la formación inicial.

Respecto a los cambios de los futuros profesores cuando participan en programas de formación inicial, los datos no son muy esperanzadores. Parece que, en general, se presentan resistencias importantes al cambio (PETERSON; TREAGUST, 1998), no siendo fácil conseguir que se desarrollen los modelos metodológicos recomendados desde la investigación didáctica (SKAMP; MULLER, 2001a). No obstante, algunos cambios que se detectan, al menos en una parte importante de los futuros profesores investigados, son:

a) ampliación del tipo de actividades que se tienen en cuenta (HAEFNER; ZEMBAL-SAUL, 2004);

b) valoración positiva del uso de problemas o preguntas (HAEFNER; 
ZEMBAL-SAUL, 2004);

c) consideración más activa de los estudiantes (se les anima a explorar los materiales, a intentar descubrir las ideas, etc.) (SKAMP; MULLER, 2001a);

d) Consideración didáctica de las ideas de los alumnos, aunque en un sentido bastante limitado: cuando reproducen lo que el profesor quiere escuchar, para crear un mejor ambiente de clase (MEYER et al., 1999; HAEFNER; ZEMBAL-SAUL, 2004);

e) aceptación de que puede haber más de un respuesta a los problemas y más de un resultado en los experimentos (SKAMP; MULLER, 2001a).

\section{Diseño metodológico}

La metodología seleccionada para esta investigación es de carácter cualitativo dentro del paradigma crítico. La finalidad de este tipo de investigaciones es principalmente la comprensión de cómo los sujetos experimentan, perciben e interpretan la realidad y su actuación en ella, estudiando el fenómeno en el contexto en el que se produce.

La investigación se centra en el estudio de una propuesta formativa (el curso CAIC) que se desarrolla en cinco escenarios diferentes, en los que son seleccionados cinco equipos que configuran los cinco casos analizados. El programa formativo tiene un contexto temporal definido, pues se desarrolla a lo largo del curso escolar. Es un estudio de casos de naturaleza evaluativa, ya que intenta analizar/valorar los resultados de una intervención específica, vinculados a la progresión de las ideas de los participantes.

En las páginas siguientes caracterizamos la investigación realizada, el contexto donde se ha desarrollado, los instrumentos para la obtención de los datos y el procedimiento de análisis empleado.

\subsection{Problema de investigación y sistema de categorías}

El problema central de la investigación es:

a) ¿cómo progresan las concepciones de diversos equipos de profesores en formación en relación con la metodología cuando participan en un 
curso de orientación constructivista (y qué obstáculos aparecen)?

El estudio de este problema nos permite:

- describir y analizar la progresión de las concepciones sobre la metodología de enseñanza,

- inferir los obstáculos didácticos y epistemológicos que bloquean su evolución,

- detectar las actividades formativas que parecen más relacionadas con los momentos de mayor progreso.

Como ya hemos dicho, para abordar el problema formulado hemos seleccionado una de las categoría de análisis: Criterio de organización de las actividades, que hemos formulado en tres niveles de progresiva complejidad, como resultado de nuestra reflexión teórica, de la experiencia en la formación del profesorado, de los modelos didácticos $\mathrm{y}$ formativos en los que venimos trabajando y del contraste con los primeros datos empíricos (PORLÁN et al., 1996; 1998; PORLÁN; RIVERO, 1998; AZCARATE, 1999b; HARRES et al., 2005).

Los niveles responden a lo siguiente:

a) el primero refleja nuestra previsión sobre las concepciones de los participantes al inicio del curso (N1);

b) el segundo describe las concepciones que consideramos que son posibles alcanzar al final de un curso de estas características (N2);

c) el tercero responde al referente teórico que nosotros consideramos deseable (N3).

Por tanto se trata de un posible Itinerario de Progresión de las concepciones de los futuros profesores en relación con la categoría estudiada, que incluye los valores inicial, posible y de referencia (CUADRO1). Como veremos más adelante, este itinerario de progresión, en interacción con los datos empíricos completos, se enriqueció con niveles intermedios. 
CUADRO 1

Itinerario de Progresión

\begin{tabular}{|c|c|c|c|}
\hline Categoria & Nivel 1 & Nivel 2 & Nivel 3 \\
\hline $\begin{array}{c}\text { Criterio de } \\
\text { organización } \\
\text { de las } \\
\text { actividades }\end{array}$ & $\begin{array}{c}\text { La secuencia } \\
\text { Metodológica } \\
\text { viene } \\
\text { determinada } \\
\text { por la } \\
\text { lógica de los } \\
\text { contenidos y } \\
\text { pretende su } \\
\text { transmisión a } \\
\text { los alumnos. }\end{array}$ & $\begin{array}{l}\text { La secuencia } \\
\text { metodológica tiene } \\
\text { relación con } \\
\text { las ideas de } \\
\text { los alumnos y } \\
\text { pretende, de } \\
\text { manera más o } \\
\text { menos explícita, } \\
\text { ampliarlas, } \\
\text { corregirlas y } \\
\text { sustituirlas por } \\
\text { el conocimiento } \\
\text { verdadero. }\end{array}$ & $\begin{array}{c}\text { La secuencia } \\
\text { metodológica tiene } \\
\text { relación con las ideas } \\
\text { de los alumnos y } \\
\text { pretende } \\
\text { favorecer su } \\
\text { evolución a } \\
\text { través de procesos de } \\
\text { investigación } \\
\text { (planteamiento de } \\
\text { problemas, } \\
\text { formulación } \\
\text { de hipótesis, } \\
\text { contraste con otras } \\
\text { informaciones, } \\
\text { establecimiento de } \\
\text { conclusiones y } \\
\text { reflexión } \\
\text { sobre lo aprendido). } \\
\text { Los contenidos se } \\
\text { consideran } \\
\text { como herramientas } \\
\text { para abordar dichos } \\
\text { problemas }\end{array}$ \\
\hline
\end{tabular}

\subsection{Contexto formativo y muestra}

El curso (CAIC) responde a un referente epistemológico constructivista, según el cuál las estrategias basadas en la investigación son especialmente adecuadas tanto para la enseñanza de los alumnos como para la formación de los profesores. Como hemos dicho en la introducción, el curso se organiza en torno a tres PPP:

a) PPP I: las ideas espontáneas de los alumnos (naturaleza, cambio, detección y análisis);

b) PPP II: los contenidos escolares (fuentes, tipos, relaciones, niveles de formulación y manera de presentarlos a los alumnos); 
c) PPP III: la metodología de enseñanza (concepto y sentido de la actividad, secuencia metodológica y recursos didácticos).

El curso comienza con el tratamiento de las ideas de los alumnos porque, como señalan varios autores, es una herramienta de primer orden para transformar el conocimiento de los futuros profesores (JONES; CARTER; RUA, 1999; MORRISON; LEDERMAN, 2003). Empezar a conocer y a tener en cuenta las ideas de los alumnos les ayuda a distanciarse del modelo tradicional de enseñanza.

La metodología de formación, por tanto, gira en torno a la participación activa de los futuros profesores en la construcción de su conocimiento profesional. En líneas generales esta metodología se caracteriza por fomentar el análisis, la reflexión crítica, la discusión en pequeños grupos de trabajo y el debate entre todos los participantes. El papel del formador es de mediador y orientador: revisa los informes, interviene en los debates abriendo interrogantes, facilita documentos que favorezcan el contraste de ideas y la reflexión, orienta las puestas en común y facilita las síntesis finales.

En el PPP I, los futuros profesores, organizados en equipos, deben investigar las ideas de 50 alumnos de Educación Primaria (6 a 12 años) sobre un contenido del curriculo de ciencias o matemáticas escogido libremente por ellos según sus intereses (actividades 1 a 6).

En el PPP II, deben diseñar los contenidos que consideran adecuados en relación a la temática escogida y teniendo en cuenta las ideas de los alumnos investigadas anteriormente (actividades de la 7 a la 9).

Por último para tratar el PPP III, deben diseñar el plan de actividades que consideran adecuado para hacer evolucionar las ideas de los alumnos acerca de los contenidos seleccionados. Los datos que se presentan y analizan en este artículo proceden fundamentalmante de las producciones escritas elaboradas por los futuros profesores durante el tratamiento de este problema profesional (actividades de la 10 a la 12).

Pero, veamos más en detalle el desarrollo de cada una de estas tres actividades:

a) actividad 10. Los equipos elaboran una primera propuesta de 
actividades para enseñar los contenidos seleccionados. Lo hacen sin consultar ningún material, teniendo en cuenta solo sus ideas en ese momento. Los equipos exponen sus producciones en la clase y se analizan entre todos en relación con: las actividades que se proponen (su papel y su sentido); cómo están organizadas (cómo se ordenan y secuencian, y por qué) y los recursos didácticos que se usan (su papel y su sentido); b) actividad 11. A continuación se presentan, analizan y debaten otras propuestas de actividades obtenidas de experiencias de innovación e investigación didáctica. En estas propuestas las actividades y los recursos didácticos juegan distintos papeles y las secuencias se establecen según la metodología que inspira cada experiencia. Posteriormente, se leen y analizan textos que presentan diferentes modelos metodológicos generales y los argumentos didácticos que los fundamentan, fundamentalmente ejemplificaciones y textos teóricos coherentes con metodologías basadas en la investigación de los alumnos. Finalmente, los equipos cumplimentan un Guión de Reflexión que les ayuda a relacionar su primera propuesta de actividades con los ejemplos alternativos presentados;

c) actividad 12. Por último, los equipos reconsideran su propuesta inicial y elaboran una segunda, justificando los cambios introducidos, que someten a crítica ante el resto de los participantes en el curso.

Como se observa, cada uno de los tres problemas se aborda a través de una secuencia de actividades formativas organizadas en tres momentos (CUADRO 2):

a) inicial, para activar, tomar conciencia y elaborar los puntos de vista de los participantes sobre el problema planteado;

b) intermedio, para provocar contraste y reflexión argumentada entre esos puntos de vista y otras informaciones procedentes de diferentes fuentes. Estas informaciones se seleccionan tomando como referencia el nivel de partida de los sujetos;

c) final, para facilitar la reelaboración de los puntos de vista iniciales y la reflexión sobre los cambios que se hayan producido. 
CUADRO 2

Actividades desarrolladas en el PPP III

\begin{tabular}{l|ll}
\hline & \multicolumn{2}{c}{ Secuencia formativa } \\
\cline { 2 - 3 } $\begin{array}{l}\text { ¿Qué secuencia } \\
\text { de actividades } \\
\text { puede favorecer } \\
\begin{array}{l}\text { que evolucionen } \\
\text { las ideas de los } \\
\text { alumnos? }\end{array}\end{array}$ & Momento inicial & $\begin{array}{l}\text { A.10: Elaborar una primera versión } \\
\text { de la secuencia de actividades }\end{array}$ \\
& Momento intermedio & $\begin{array}{l}\text { A.11: Conocer y debatir } \\
\text { aportaciones de la investigación } \\
\text { didáctica. Guión de reflexión }\end{array}$ \\
& Momento final & $\begin{array}{l}\text { A.12: Elaborar la segunda versión } \\
\text { de la secuencia de actividades }\end{array}$ \\
\hline
\end{tabular}

El CAIC se desarrolló durante un semestre en distintas asignaturas de la Formación Inicial del Profesorado, en diferentes universidades y conducidos por distintos profesores.

Las asignaturas previas al desarrollo de este curso están relacionadas con la enseñanza de algunas materias escolares (matemáticas, ciencias de la naturaleza, etc.) o con la enseñanza de contenidos psicopedagógicos generales; en ningún caso, los participantes han realizado Prácticas de Enseñanza.

El número de futuros profesores que cursaron cada asignatura varía desde 37 hasta 50, la mayoría de ellos mujeres y con una edad de entre 18 y 25 años.

Normalmente han cumplimentado estudios de Bachillerato antes de acceder a la universidad, sin que predomine ninguna de las especialidades (ciencias, arte, tecnología o humanidades). Estos estudiantes se organizaron en equipos de entre 4 y 6 miembros, formándose entre 9 y 10 equipos en cada contexto.

Se toma como unidad informativa los equipos y el trabajo desarollado por ellos. El papel del trabajo colaborativo y la elaboración del conocimiento compartido, como piezas claves del desarrollo profesional, son referentes teóricos que justifican esta elección (TILLEMA, 2000; WATTERS; GINNS, 2000; OSBORNE; COLLINS, 2001; TILLEMA; VAN DER WESTHUIZEN, 2006; ZELLERMAYER; TABAK, 2006).

La muestra seleccionada como objeto de estudio está constituida por 5 casos (A, B, C, D y E), correspondientes a un equipo de cada contexto en que se desarrolló el curso CAIC. Los equipos que constituyen la 
muestra se escogieron al azar de entre todos los equipos formados en cada contexto. En el CUADRO 3 se detallan las características de esta muestra.

CUADRO 3

Muestra

\begin{tabular}{|c|c|c|c|c|c|}
\hline Caso & Temática & Universidad & $\begin{array}{l}\text { Estudios } \\
\text { (Program) }\end{array}$ & $\begin{array}{l}\text { Asignatura } \\
\text { (Course) }\end{array}$ & $\begin{array}{l}\mathrm{N}^{\mathrm{o}} \mathrm{de} \\
\text { alumnos/ } \\
\text { equipos }\end{array}$ \\
\hline A & $\begin{array}{l}\mathrm{La} \\
\text { reproducción } \\
\text { humana }\end{array}$ & $\begin{array}{l}\text { Universidad } \\
\text { Complutense } \\
\text { deMadrid } \\
\text { (España) }\end{array}$ & $\begin{array}{c}\text { Diplomado } \\
\text { en Maestro de } \\
\text { Primaria }\end{array}$ & $\begin{array}{l}\text { Concepciones } \\
\text { de los alumnos } \\
\text { sobre la ciencia } \\
\text { (Optativa. } 40 \\
\text { horas. } 1^{\circ} \text { curso, } \\
2^{\circ} \text { semestre) }\end{array}$ & $\begin{array}{l}37 \text { estudiantes } \\
\text { (9 equipos) }\end{array}$ \\
\hline B & Los animales & $\begin{array}{l}\text { Universidad de } \\
\text { Sevilla (España) }\end{array}$ & $\begin{array}{c}\text { Diplomado } \\
\text { en Maestro } \\
\text { de Educación } \\
\text { Especial }\end{array}$ & $\begin{array}{l}\text { Didáctica Del } \\
\text { área de } \\
\text { Conocimiento } \\
\text { del Medio } \\
\text { (Obligatoria. } 50 \\
\text { horas. } 1^{\circ} \text { curso, } \\
2^{\circ} \text { semestre) }\end{array}$ & $\begin{array}{l}57 \text { estudiantes } \\
\text { (10 equipos) }\end{array}$ \\
\hline $\mathrm{C}$ & Ácidos y bases & $\begin{array}{c}\text { Centro } \\
\text { Universitario- } \\
\text { UNIVATES } \\
\text { (Lajeado, Brasil) }\end{array}$ & $\begin{array}{c}\text { Licenciatura en } \\
\text { Profesor de } \\
\text { Ciencias }\end{array}$ & $\begin{array}{l}\text { Laboratorio de } \\
\text { Enseñanza de } \\
\text { Ciencias I } \\
\text { (Obligatoria. } 40 \\
\text { horas. } 1^{\circ} \text { curso, } \\
2^{\circ} \text { semestre) }\end{array}$ & $\begin{array}{l}40 \text { estudiantes } \\
\text { (10 equipos) }\end{array}$ \\
\hline D & $\begin{array}{l}\text { La Tierra y el } \\
\text { Universo }\end{array}$ & $\begin{array}{l}\text { Universidad de } \\
\text { Sevilla (España) }\end{array}$ & $\begin{array}{c}\text { Diplomado } \\
\text { en Maestro de } \\
\text { Primaria }\end{array}$ & $\begin{array}{l}\text { Ciencias de la } \\
\text { Naturaleza } \\
\text { y su } \\
\text { Didáctica } \\
\text { (Obligatoria. } 80 \\
\text { horas. } 2^{\circ} \text { curso, } \\
1^{\circ} \text { semestre) }\end{array}$ & $\begin{array}{c}53 \text { estudiantes } \\
\text { (9 equipos) }\end{array}$ \\
\hline E & Los polígonos & $\begin{array}{l}\text { Universidad de } \\
\text { Cádiz (España) }\end{array}$ & $\begin{array}{c}\text { Diplomado } \\
\text { en Maestro de } \\
\text { Primaria }\end{array}$ & $\begin{array}{l}\text { Desarrollo del } \\
\text { pensamiento } \\
\text { matemático } \\
y \text { su } \\
\text { Didáctica } \\
\text { (Obligatoria. } 60 \\
\text { horas. } 1^{\circ} \text { curso, } \\
1^{\circ} \text { semestre) }\end{array}$ & $\begin{array}{c}52 \text { estudiantes } \\
\text { (9 equipos) }\end{array}$ \\
\hline
\end{tabular}




\subsection{Instrumentos y técnicas de análisis}

Los datos se obtuvieron en los tres momentos del curso mencionados anteriormente: inicial (al elaborar las primeras concepciones sobre el problema), intermedio (al someterlas a contraste) y final (al re-elaborarlas de manera argumentada). Los instrumentos de recogida de información fueron algunos de los documentos producidos por los equipos de la clase, y que se recogen en el CUADRO 4.

CUADRO 4

Instrumentos de recogida de información

\begin{tabular}{c|ccc}
\hline \multirow{4}{*}{ Instrumentos } & M. Inicial & M. Intermedio & M. final \\
\cline { 2 - 4 } & $\begin{array}{c}\text { Primera propuesta } \\
\text { de la secuencia de } \\
\text { actividades } \\
\text { (Documento 10) }\end{array}$ & $\begin{array}{c}\text { Guión de reflexión } \\
\text { sobre cómo enseñar }\end{array}$ & $\begin{array}{c}\text { Segunda propuesta } \\
\text { de la secuencia de } \\
\text { actividades } \\
\text { (Documento 11) }\end{array}$ \\
\hline
\end{tabular}

Los documentos 10 y 12 son producciones libres, en las que la reflexión en torno al problema es totalmente abierta, sin restricciones de ningún tipo (Actividades 10 y 12). El documento 11 es un guión de reflexión que actúa como cuestionario de preguntas abiertas que dirigen la discusión y la toma de decisiones en los equipos (Actividad 11). Las argumentaciones presentadas en estos documentos no sólo organizan el aprendizaje de los grupos sino que nos dan claves para comprender sus concepciones y su posible evolución. Los documentos 10, 11 y 12 se pueden ver en el anexo 2.

La información obtenida fue convertida en unidades de información y codificada según la categoría $(1,2,3)$, el caso/equipo $(A, B, C, D$, E), el documento $(10,11,12)$ y el número de orden de aparición en el documento. Para el tratamiento de estos datos se ha seguido el siguiente procedimiento:

a) identificación de Unidades de Información Significativas (UIS) en las producciones escritas; 
b) codificación y clasificación de las mismas en la categoría correspondiente. Elaboración de Proposiciones de Síntesis (PS) a partir de las diferentes UIS para cada categoría, momento formativo y caso, con un bajo nivel de inferencia;

c) validación de las categorizaciones y de la elaboración de proposiciones a través de un proceso de triangulación en el que cada caso fue revisado independientemente por un mínimo de dos participantes en la investigación. Hubo un índice de concordancia de más del $90 \%$ en todos los casos. Las divergencias fueron analizadas separadamente por todo el equipo de investigación buscando un consenso y, cuando esto no fue posible, se adoptó la posición mayoritaria.

El análisis y la discusión de los datos se ha realizado siguiendo los pasos que se describen a continuación:

a) ubicación de las PS en los niveles de progresión previstos (N1, N2 y N3). Este proceso puso en evidencia la necesidad de definir niveles intermedios (N01 y N12) para recoger mejor la diversidad presente en los datos;

b) teniendo en cuenta lo anterior, presentación del Itinerario de Progresión identificado para cada caso y categoría;

c) en cada categoría, formulación y análisis de los obstáculos que están presentes en la muestra y su relación con las actividades formativas;

d) por último, integrando los niveles definidos en cada categoría, elaboración de los Niveles Generales de Progresión sobre la metodología y los Obstáculos Generales asociados.

Un ejemplo de una parte sustancial de todo este procedimiento de tratamiento y análisis de los datos puede verse en el CUADRO 5. 


\section{CUADRO 5}

Procedimiento de tratamiento y análisis de los datos

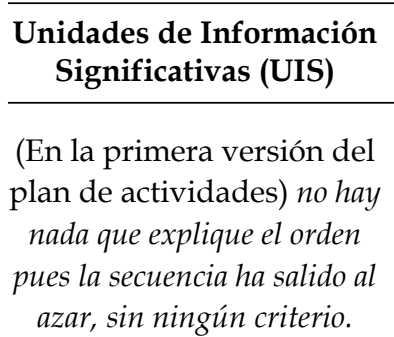

(El modelo metodológico que defendemos en estos momentos es:) 1. Problema.

2. Ideas previas. 3. Nuevas

informaciones. 4 Actividades (motivación, aplicación, intervención en el medio). 5. Actividades de refuerzo.

Debemos partir del planteamiento de un problema que esté dentro del contexto cotidiano de los niños, es decir, que les resulte familiar. Una vez establecido el problema, podemos ver que ideas tienen los alumnos sobre el mismo $y$, poco a poco, irnos acercando más a ellos. Gracias a estas ideas previas nos será más fácil seleccionar las informaciones que debemos darles, para que les sea más fácil relacionar ambas. Como siguiente punto creemos conveniente

\begin{tabular}{c|c}
$\begin{array}{c}\text { Proposición de } \\
\text { Síntesis (PS) }\end{array}$ & Nivel
\end{tabular}

En nuestra primera versión del plan de actividades no hay nada que explique el orden, la secuencia se ha diseñado

al azar, sin ningún criterio. No se tuvieron en cuenta las ideas de los alumnos, ni se plantearon problemas, ni se siguió un orden en el planteamiento de la metodología, sólo se elaboraron ejercicios prácticos.

Se deben introducir cambios para mejorarla: plantear problemas y averiguar las ideas de los alumnos para poder facilitar que los alumnos relacionen dichas ideas con lo que le queremos enseñar.

La secuencia

metodológica que en estos momentos consideramos adecuada es:

1. Planteamiento de un problema del contexto cotidiano de los niños, que les resulte familiar.

2. Ideas previas. Podemos ver qué ideas tienen los
La secuencia metodológica tiene relación con las ideas de los alumnos y pretende, de manera más o menos explícita, ampliarlas, corregirlas y sustituirlas por el conocimiento verdadero.

(Nivel 2) 
programar actividades tanto de motivación, como de aplicación y de intervención en el medio. Con ellas evaluaremos a los alumnos para ver si han entendido lo que les hemos explicado anteriormente. Si se hacen las actividades y hay algunos alumnos que no se han enterado, debemos de poner más actividades de refuerzo.

No tiene coherencia (nuestro nuevo plan de enseñanza respecto a la primera versión), puesto que en la primera versión ni hemos tenido en cuenta las ideas de los alumnos ni hemos seguido un orden en el planteamiento de la metodología, sólo habíamos elaborado ejercicios prácticos.

(Con respecto a nuestra primera versión) un primer cambio (que debemos hacer), es que en la primera versión no hemos tenido en cuenta el planteamiento de problemas, ni averiguar sus ideas previas. alumnos y poco a poco irnos acercando más a ellos.

3. Nueva información que, gracias a los anteriores pasos, nos será más fácil decidirla. Por la misma razón, a los alumnos les será más fácil relacionarla con sus ideas.

4. Actividades prácticas de motivación, aplicación e intervención en el medio. Con ellas se evaluará si los alumnos han entendido lo explicado anteriormente.

5. Actividades de refuerzo para los alumnos que lo necesiten.

Como vemos en este ejemplo, hemos identificado cinco UIS (todas ellas procedentes del documento 11 de uno de los equipos) y desde ellas elaboramos la PS correspondiente a una de las categorías (criterio de organización de las actividades) en uno de los momentos del curso (intermedio). 


\section{Resultados y discusión}

En la categoría "Criterio de organización de las actividades" no se da la progresión que esperábamos del nivel N1 al nivel N2 (ver Figura 1). De hecho, sólo uno de los equipos (caso A) organiza, en el momento inicial, la secuencia metodológica en función de la lógica de los contenidos y con el objeto de facilitar su transmisión a los alumnos. El caso E parte de una situación parecida (N12), pues el eje organizador de la secuencia también son los contenidos pero pretendiendo que los alumnos los descubran por sí mismos. El resto de los equipos $(\mathrm{B}, \mathrm{C}$ y $\mathrm{D})$ parten de niveles que hemos considerado más simples, pues no organizan con ningún criterio las actividades propuestas (N01), o más complejos, que organiza la secuencia en función de las ideas de los alumnos. Como en la categoría anterior, los datos iniciales nos han permitido definir dos niveles nuevos que no aparecían en el itinerario de progresión previsto (N01 y N12).

\section{CUADRO 6}

Criterio de organización de las actividades

\begin{tabular}{|c|c|c|c|}
\hline \multicolumn{2}{|c|}{ Niveles detectados } & Antes & Durante \\
\hline N3 & $\begin{array}{c}\text { La secuencia } \\
\text { metodológica tiene } \\
\text { relación con las ideas } \\
\text { de los alumnos y } \\
\text { pretende favorecer su } \\
\text { evolución a través de } \\
\text { procesos de investigación } \\
\text { (planteamiento de } \\
\text { problemas, formulación } \\
\text { de hipótesis, contraste } \\
\text { con otras informaciones, } \\
\text { establecimiento de } \\
\text { conclusiones y reflexión } \\
\text { sobre lo aprendido). Los } \\
\text { contenidos se consideran } \\
\text { como herramientas } \\
\text { para abordar dichos } \\
\text { problemas. }\end{array}$ & & E A \\
\hline
\end{tabular}




\begin{tabular}{|c|c|c|c|}
\hline \multicolumn{2}{|c|}{ Niveles detectados } & Antes & Durante \\
\hline N2 & $\begin{array}{l}\text { La secuencia metodológica } \\
\text { tiene relación con las ideas } \\
\text { de los alumnos y pretende, } \\
\text { de manera más o menos } \\
\text { explícita, ampliarlas, } \\
\text { corregirlas y sustituirlas por } \\
\text { el conocimiento verdadero. }\end{array}$ & $\mathrm{D}$ & D B C \\
\hline N12 & $\begin{array}{l}\text { La secuencia metodológica } \\
\text { viene determinada por la } \\
\text { lógica de los contenidos y } \\
\text { persigue ampliar las ideas } \\
\text { de los alumnos, corregir } \\
\text { los errores conceptuales } \\
\text { y sustituirlos por el } \\
\text { conocimiento verdadero. }\end{array}$ & $\mathrm{E}$ & \\
\hline N1 & $\begin{array}{l}\text { La secuencia metodológica } \\
\text { viene determinada por la } \\
\text { lógica de los contenidos y } \\
\text { pretende su transmisión a } \\
\text { los alumnos. }\end{array}$ & A & \\
\hline
\end{tabular}

Veamos las progresiones detectadas. Entre el momento inicial y el intermedio, los equipos A y E experimentan una evolución muy importante, pues pasan de organizar la secuencia en función de los contenidos a organizarla para favorecer la evolución de las ideas de los alumnos mediante procesos de investigación (N3). Sin embargo, en el momento final estos dos equipos experimentan una regresión, como ocurrió en la categoría anterior, desde el nivel 3 hasta el nivel 2. La secuencia de actividades propuesta finalmente pretende sustituir las ideas iniciales de los alumnos por las consideradas correctas por el profesor, como se observa, por ejemplo, en la secuencia diseñada por el equipo A:

Caso A:

El orden [de las situaciones propuestas en el plan de enseñanza] es: primero hacer la distinción entre aparato reproductor masculino y 
femenino, utilizando el busto desmontable. Segundo, explicación de las funciones de cada órgano mediante el busto, películas y comparación con objetos de la vida cotidiana. Tercero, explicación del concepto de fecundación mediante esquema o comparación con animales. (Momento inicial; Documento 10)

El nuevo plan de enseñanza debe estar constituido por: Actividades de motivación: ofrecer al niño propuestas de investigación sobre algo cercano a él.

Actividades de puesta en común: con el resto de los compañeros sobre el tema de investigación (así intercambian ideas previas con sus compañeros).

Actividades experimentales: a partir de las cuales se acercan a la realidad.

Actividades de explicación del profesor: enlazan los conocimientos previos con los nuevos, poniendo en relación todo lo realizado desde que comenzó la actividad de motivación, las ideas que aportan los alumnos en el debate y demostrándoles lo que han aprendido a través de su investigación, sugiriéndoles ideas, etc.

Actividades de aplicación de lo aprendido: realizar ejercicios de aplicación que, además, sirvan para comparar las ideas que tenían antes y las que han elaborado a lo largo de todo el proceso.

Actividades de puestas en común: para que comparen lo que han aprendido entre sí, que aporten y que lleguen a una conclusión que responda a la actividad de partida y también que puedan justificar si les ha servido de algo el proceso. (Momento intermedio; Documento 11) $1^{\circ}$ Se pregunta a los alumnos "¿Sabéis lo que pasa entre dos adultos cuando quieren tener hijos?". Se pide que cada grupo (de 3 alumnos) llegue a una conclusión, teniendo en cuenta lo que saben o han escuchado fuera de clase. Se hace una puesta en común en forma de debate. El profesor actúa de observador y modificador apuntando en la pizarra las posibles respuestas. Al finalizar el debate, el profesor anunciará a los alumnos que las conclusiones iniciales quedarán en un mural que se colgará en la pared del aula, para revisarlas al terminar el tema de la reproducción. 
$2^{\circ}$ Se les propone que, en grupos de 3 , hagan una encuesta para investigar las causas por las que se tienen hijos. Cuestión: ¿Por qué nace un bebé? Esta encuesta la aplicarán a familiares, vecinos, etc...

$3^{\circ}$ Cuando los alumnos hayan realizado sus encuestas, se les pedirá que recopilen la información en forma de gráfico de barras. Se procederá a realizarlo en grupos de 3 (como inicialmente)...

$4^{\mathrm{o}}$. Tras reconocer que en la reproducción intervienen 2 sexos, trataremos de que los alumnos identifiquen las diferencias entre ellos. Para eso acudimos al laboratorio y les ofrecemos bustos de los diferentes órganos sexuales, para que la manipulación ayude a la interiorización del contenido a enseñar. Se les dará un tiempo aproximado de 10 minutos para que, de forma libre y espontánea, observen las diferencias...

$5^{\circ}$. Ya en el aula se procederá a trabajar en grupo (de 3 en 3 , los iniciales). El profesor ofrecerá a los alumnos los diferentes órganos (masculino y femenino) en forma de recortables. Trabajarán con ellos pintándolos y construyendo un mural de cada uno de los aparatos (en cada cartulina ya estaba dibujada la silueta). El profesor se prestará a dar las informaciones y aclaraciones que sean necesarias a los alumnos, así como a resolver dudas, solucionar conflictos, etc., siempre y cuando sean los alumnos quienes pidan la ayuda. (Momento final; Documento 12)

Como podemos observar, este equipo proponía en el momento intermedio una secuencia de actividades basada en la investigación de los alumnos, a lo largo de la cual podían acercarse a la realidad, enlazar los conocimientos nuevos con los previos, comparar lo que han aprendido entre sí,... etc. Sin embargo, en la secuencia diseñada en el momento final, se observa que los futuros profesores dan un valor significativo a constatar las ideas correctas de los alumnos.

Los equipos $\mathrm{B}$ y $\mathrm{C}$, que en el momento inicial no siguen ningún criterio para organizar las actividades (no hay nada que explique el orden pues la secuencia que diseñamos salió al azar, sin ningún criterio; sólo nos hemos basado en proponer muchas actividades prácticas, porque son actividades que a los niños les gustan mucho; caso B, Documento 11), en el momento intermedio proponen organizar la secuencia de actividades en función de las ideas de los alumnos. Ambos equipos comparten también, de manera más o 
menos explícita, que la finalidad de esta secuencia es sustituir las ideas de los alumnos por el conocimiento verdadero (N2).

Caso C:

Nuestro modelo metodológico en este momento es: - Ideas previas: conocimiento existente del contenido que va a ser estudiado; - Explicar los errores: corregir ideas distorsionadas, incrementar el conocimiento, comprender los contenidos con debates y prácticas; - Test: verificar si ha habido crecimiento en el contenido tratado. (Momento intermedio; Documento 11)

Por último, el equipo D se ha mantenido en el mismo nivel en los momentos inicial, intermedio y final, precisamente el que considerábamos que era posible alcanzar al final del proceso formativo (N2). Desde el inicio, su propuesta está ya más centrada en los alumnos que en el profesor, pues su secuencia metodológica toma como eje las ideas de los alumnos. Como ellos mismos declaran en el momento intermedio, esta consideración de las ideas de los alumnos se hace para poder corregirlas.

Caso D:

Vamos a desarrollar nuestra unidad didáctica a través de una serie de preguntas/problemas o proyectos, en los cuales vamos a seguir siempre los mismos pasos: 1) Se lanza la pregunta/problema o proyecto y se reune a los alumnos en grupo para que hagan una puesta en común sobre el tema sin consultar nada. 2) Se va poniendo en la pizarra las opiniones de todos los grupos y una vez terminado, el profesor, mediante nuevas cuestiones, hará reflexionar a los alumnos sobre aquellas cosas que falten o estén mal planteadas. 3) Se dará tiempo a los niños para que investigen por su cuenta, consultando libros $u$ otros recursos, o preguntando a sus mayores. 4) En clase volverá a hacer una puesta en común sobre lo investigado por cada niño y así tendremos todos los contenidos que queremos trabajar. 5) Por último, se hará una actividad para poner en práctica todo lo aprendido y que será la única que difiera en el desarrollo 
de una pregunta/problema o proyecto respecto a otra (Momento inicial; Documento 10)

En la primera versión del plan de enseñanza el orden de las actividades viene condicionado por: las ideas de los alumnos, en el sentido de que, grosso modo, se sigue una secuencia del tipo: conocer las ideas de los alumnos, dar información para corregir los errores y vacíos detectados y comprobar si las ideas han cambiado.

El modelo metodológico que hemos hecho ahora es prácticamente el mismo que el de la primera versión, la única diferencia es que una de las actividades finales no está encuadrada en una pregunta o proyecto concreto, sino que es general para todo el temario. (Momento intermedio; Documento 11)

Trabajo con el primer problema: 1. Se lanza la siguiente pregunta: ¿Siempre ha sido igual el universo? Los alumnos se reúnen en grupo para hacer una puesta en común sobre el tema sin consultar nada. 2. Se van poniendo en la pizarra las opiniones de todos los grupos y, una vez terminado, el profesor, mediante cuestiones, hace reflexionar a los alumnos sobre aquellas cosas que falten o estén mal planteadas. 3. Se da tiempo a los niños para que investiguen por su cuenta, consultando libros, otros recursos o preguntando a sus mayores. 4 . En clase se vuelve a hacer una nueva puesta en común sobre lo investigado y así tendremos todos los contenidos que queremos trabajar. 5. Se hace también una actividad final para aplicar todo lo aprendido: hacemos un debate sobre este asunto.

Trabajo con el segundo problema: 1. Se lanza la siguiente pregunta: ¿Qué hay en el universo? Los alumnos se reúnen en grupo para hacer una puesta en común sobre el tema sin consultar nada. 2. Se van poniendo en la pizarra las opiniones de todos los grupos y, una vez terminado, el profesor, mediante cuestiones, hace reflexionar a los alumnos sobre aquellas cosas que falten o estén mal planteadas. 3. Se da tiempo a los niños para que investiguen por su cuenta, consultando libros, otros recursos o preguntando a sus mayores. 4 . En clase se vuelve a hacer una nueva puesta en común sobre lo investigado y así tendremos todos los contenidos que queremos trabajar. 5. Se hace también una actividad final para aplicar todo lo aprendido: reunir a los niños una noche en el patio 
del colegio para observar el cielo. (...). (Momento final; Documento 12) Como vemos, aunque este equipo incorpora elementos importantes de una metodología basada en la investigación de los alumnos planteamiento de preguntas, formulación de hipótesis, contraste con otras informaciones, etc.-, cree que la búsqueda de información que se propone a los alumnos será tan fructífera que les permitirá aprender todos los contenidos que queremos trabajar.

En definitiva, podemos decir que, salvo en un caso, en el que no se detectan cambios, existe progresión entre el momento inicial y final. Las progresiones identificadas son de cierta relevancia, pues se ha pasado, en general, de una secuencia metodológica desorganizada u organizada en función de los contenidos, a una secuencia metodológica organizada en función de las ideas de los alumnos. Las distintas situaciones que se han dado a lo largo del proceso (progresiones, no cambio, regresiones) han reagrupado a los equipos en un mismo nivel (N2), precisamente el nivel que considerábamos posible.

Parece, pues, confirmarse nuestra impresión de que aunque se le dé importancia a los alumnos y se adopten metodologías que tenga como eje sus ideas, es bastante difícil asumir que dichas ideas pueden cambiar y que los alumnos pueden elaborar significados intermedios entre sus puntos de partida y las nuevas informaciones. Esto está relacionado, a nuestro entender, con dos obstáculos importantes, uno de carácter didáctico y otro más profundo de naturaleza epistemológica. El primero tiene que ver con la idea de que la enseñanza es la causa directa del aprendizaje, por lo que se espera que los alumnos aprendan fielmente lo que se les enseña, sin interferencia de sus ideas. El segundo, el absolutismo epistemológico, según el cual el conocimiento científico es un conocimiento verdadero y superior y, en consecuencia, el que los alumnos deben exactamente aprender.

Algunos estudios han detectado resultados similares a los nuestros. En el estudio que ya hemos mencionado de Yerrick, Parke y Nugent (1997), los participantes consideraban a los estudiantes como los receptores del conocimiento y con poca influencia en las decisiones acerca de qué y cómo enseñar. Estas visiones se modificaron tras el 
proceso formativo de manera que, al rediseñar sus lecciones de ciencia, los profesores consideraron las concepciones de los estudiantes como un elemento importante a tener en cuenta, pero sin renunciar a la idea de que existe un conjunto predeterminado de ideas verdaderas que son las que se deben enseñar y, por tanto, aprender.

Meyer et al. (1999) describen, en forma de estudio de caso, las concepciones y prácticas de tres maestros de Primaria en formación en un curso de ciencias basado en el cambio conceptual. Como en nuestro estudio, una de las maestras participantes, Karen, experimenta algunos cambios en sus concepciones sobre las ideas de los estudiantes, y sobre su papel activo en la construcción de conocimientos, pero no reconoce que el resultado final del aprendizaje pueda ser una mezcla de dichas ideas y de las nuevas, sino que espera que los alumnos las cambien por los conocimientos científicos adecuados, mediante las actividades propuestas.

En el estudio de Lemberger et al. (1999) se analiza el cambio de las concepciones de tres futuros profesores de Biología de Secundaria cuando participan en un curso basado en el cambio conceptual. Al inicio, la visión que compartían sobre la enseñanza era básicamente transmisiva y organizada en función de la lógica de los contenidos. A lo largo del curso pasaron a planteamientos más centrados en el estudiante. Todos prestaron atención a detectar las ideas previas de los alumnos, aunque luego no las tuvieron en cuenta; en este estudio el afloramiento de las ideas de los alumnos juega más bien un papel afectivo, con la intención de crear un ambiente de aula agradable. En los casos estudiados por nosotros, la detección de ideas de los alumnos ha tenido más bien el papel de detectar vacíos, errores, e ideas incompletas.

Sin embargo, en otros estudios los resultados son diferentes a los nuestros. En algunas investigaciones no se detectan apenas cambios en las secuencias de actividades que proponen los profesores tras determinados procesos formativos (GUSTAFSON; ROWELL, 1995). En otras, como la de Lauriala (1998), que estudia el cambio de las concepciones de profesores en un programa de formación basado en la asistencia a clases de colegas con visiones constructivistas, los profesores declaran al final que el aprendizaje de los estudiantes es diverso y que no siempre coincide 
con el predeterminado. Igualmente, en el estudio de Crawford (1999), una futura maestra experimenta cambios muy importantes diseñando y desarrollando en las Prácticas de Enseñanza secuencias de actividades como las que conforman nuestro nivel 3 (nivel teórico de referencia). Es destacable que en estos casos está presente un elemento que nosotros no hemos podido incluir en el curso CAIC, dadas las características organizativas de la Formación Inicial en nuestros contextos: la interacción con la práctica de aula.

\section{Conclusiones}

Podemos concluir diferenciando cuatro Niveles Generales en relación a la metodología, que se pueden ordenar en un Itinerario General de Progresión. Dichos niveles son:

a) Nivel General de INTUICIÓN (N01), que no esperábamos encontrar. En él, la metodología no responde a un modelo articulado y las situaciones de enseñanza que se proponen no se ordenan lógicamente. El equipo C, en el momento inicial, es un claro ejemplo de este nivel. El obstáculo que subyace es que parece que no es necesaria una determinada metodología para poder enseñar. Se trata de un obstáculo que aparece en nuestra muestra únicamente en el momento inicial, afectando de manera desigual a cuatro de los cinco equipos analizados. La actividad formativa que ha podido tener más influencia para su superación es la Actividad 11, en la que se somete a análisis y contraste crítico las propuestas iniciales de los equipos;

b) Nivel General de TRANSMISIÓN (N1 y N12). En él, la lógica metodológica es subsidiaria de la lógica de los contenidos que se quieren transmitir. El obstáculo que subyace es la creencia de que los alumnos y sus ideas no influyen en la metodología. Este obstáculo está presente en los momentos iniciales e intermedio de nuestro estudio, pero afecta a pocos casos y no en todas las categorías. Las actividades formativas que han podido influir más en su superación son las relacionadas con la lectura y el análisis de ejemplos de metodologías innovadoras (lectura de artículos y observación de vídeos sobre experiencias alternativas basadas en la 
investigación de los alumnos), así como el diseño del plan de enseñanza definitivo (Actividades 11 y 12);

c) Nivel General de SUSTITUCIÓN (N2). Es el que considerábamos posible alcanzar y en el que, efectivamente, se sitúan todos los casos al final del curso. Este modelo está más centrado en el alumno que en el profesor, aunque sin renunciar a que hay un saber predeterminado que hay que aprender. La metodología de enseñanza responde a una lógica basada en la detección de las ideas de los alumnos y en su ampliación o/y sustitución por el conocimiento verdadero. El obstáculo que subyace es entender que la enseñanza es la causa directa del aprendizaje. En un ámbito más profundo, está influyendo el absolutismo epistemológico. Estos obstáculos están presentes en la muestra estudiada durante todos los momentos (inicial, intermedio y final); son los que no hemos podido "sortear" con nuestra propuesta formativa. La presencia de estos obstáculos parece impedir que se opte por metodologías que faciliten la construcción de conocimientos genuinos y negociados por los alumnos. Seguramente para incidir sobre ellos es necesario participar en más ocasiones en cursos de similares características al que hemos desarrollado, o en propuestas formativas que permitan el desarrollo del diseño realizado y la reflexión sobre ello, o la participación en clases de colegas innovadores y constructivistas (GUSTAFSON; ROWELL, 1995; LAURIALA, 1998);

d) Nivel General de INVESTIGACIÓN (N3), es el nivel teórico de referencia. En él, la metodología de enseñanza responde a una lógica basada en la investigación de problemas relevantes para favorecer la evolución de las ideas de los alumnos. Dichas ideas se consideran el eje del proceso de enseñanza-aprendizaje. Dos de los cinco equipos analizados se han aproximado a este modelo en el momento intermedio, aunque sin consolidarse en él.

Este Itinerario de Progresión General puede representarse en forma de una escalera en la que podemos visualizar los cuatro Niveles Generales formulados, los Obstáculos Generales que dificultan la progresión de unos a otros y las Actividades Formativas que parecen ayudar a la progresión (Figura 2). 


\section{CUADRO 7}

Criterio de organización de las actividades

\begin{tabular}{|c|c|c|c|c|}
\hline & Niveles detectados & Antes & Durante & Después \\
\hline N3 & $\begin{array}{l}\text { La secuencia metodológica tiene } \\
\text { relación con las ideas de los } \\
\text { alumnos y pretende favorecer su } \\
\text { evolución a través de procesos de } \\
\text { investigación (planteamiento de } \\
\text { problemas, formulación de hipótesis, } \\
\text { contraste con otras informaciones, } \\
\text { establecimiento de conclusiones y } \\
\text { reflexión sobre lo aprendido). Los } \\
\text { contenidos se consideran como } \\
\text { herramientas para abordar dichos } \\
\text { problemas. }\end{array}$ & & $E$ & \\
\hline N2 & $\begin{array}{l}\text { La secuencia metodológica tiene } \\
\text { relación con las ideas de los alumnos } \\
\text { y pretende, de manera más o menos } \\
\text { explícita, ampliarlas, corregirlas y } \\
\text { sustituirlas por el } \\
\text { conocimiento verdadero. }\end{array}$ & $\mathrm{D}$ & B & $\begin{array}{l}\text { E } \\
\text { A } \\
\text { D } \\
\text { B } \\
\text { C }\end{array}$ \\
\hline N12 & $\begin{array}{l}\text { La secuencia metodológica viene } \\
\text { determinada por la lógica de los } \\
\text { contenidos y persigue ampliar las } \\
\text { ideas de los alumnos, corregir los } \\
\text { errores } \\
\text { conceptuales y sustituirlos por el } \\
\text { conocimiento verdadero. }\end{array}$ & & & \\
\hline N1 & $\begin{array}{c}\text { La secuencia metodológica viene } \\
\text { determinada por la lógica de los } \\
\text { contenidos y pretende su transmisión } \\
\text { a los alumnos. }\end{array}$ & & & \\
\hline N01 & $\begin{array}{l}\text { La secuencia metodológica } \\
\text { es aditiva. }\end{array}$ & $\mathrm{B}$ & & \\
\hline
\end{tabular}




\section{Implicaciones formativas}

Los datos obtenidos confirman la idea de que el cambio en las ideas y en las prácticas del profesorado es un proceso que se produce de forma lenta y con grandes dificultades. La formación de profesores no puede pretender sustituir las ideas iniciales del profesorado por aquellas otras que la investigación educativa considera más adecuadas, sino que debe adoptar enfoques progresivos y constructivistas, tal como se propone para la formación del alumnado (DUIT; TREAGUST, 2003).

En relación con lo anterior, coincidimos con Flores, Gallegos \& Baroja (2000) en que es necesario moderar las expectativas de cambio en las actividades formativas y procurar transiciones progresivas, que supongan avances bien consolidados en las concepciones y prácticas de los profesores, construídos por ellos mismos, y no saltos en el vacío con pocas posibilidades de mantenerse en el tiempo.

Organizar el currículo de la formación de profesores en torno a problemas prácticos profesionales da sentido a la formación. El hecho de trabajar sobre situaciones vinculadas con la práctica futura, y reflexionar sobre como abordarlas, permite a los futuros profesores ponerse en la situación del docente y tomar decisiones, reflexionando sobre cuáles son las más adecuadas y por qué. Problematizar, por tanto, estas situaciones les hace avanzar desde sus planteamientos iniciales hacia concepciones más elaboradas.

Es imprescindible que los futuros profesores contrasten sus propias visiones con prácticas alternativas y no sólo con informaciones teóricas (DUIT; TREAGUST, 2003). No podemos olvidar que los únicos referentes prácticos directos que poseen los estudiantes son aquellos que han vivido como alumnos, es la única práctica educativa que conocen y en ella se basan, aún sin ser conscientes, para diseñar y desarrollar su enseñanza. Este referente no es contrastable con ideas teóricas generales, sino con otros de la misma naturaleza, de carácter alternativo.

Los futuros profesores necesitan experimentar sus nuevas ideas y reflexionar sobre dicha experimentación para poder ir consolidando los cambios (WATTS; JOFILI, 1998). Las reflexiones sobre el diseño de la 
práctica, que es una de las estrategias dominantes en los programas de formación, tienen influencia en las ideas de los futuros profesores, como hemos visto en nuestro estudio. Pero si esta reflexión está vinculada a la inmersión en la práctica y permite el intercambio directo con ella, creemos que es la manera más prometedora de provocar desarrollo profesional auténtico (TILLEMA, 2000; ZEMBAL-SAUL; KRAJCIK; BLUMENFELD, 2002; RUSSELL; MARTIN, 2007). Tener la oportunidad de tender un puente entre teoría y práctica es crítico para provocar el cambio en las creencias y las acciones de los futuros profesores. En esa línea, Tillema (2000) comenta que los procesos de reflexión sobre las prácticas de enseñanza y sobre las creencias que los profesores en formación activan durante las mismas, son uno de los instrumentos fundamentales para provocar un cambio o evolución estable en sus ideas. Estos procesos permiten poner en cuestión las ideas interiorizadas durante su experiencia como alumnos y construir nuevos referentes en sus prácticas como profesores.

Por último, consideramos que es necesario que los profesores desarrollen un conocimiento profesionalizado sobre la metodología de enseñanza, que les permita generar contextos de auténtico aprendizaje (y no situaciones en las que se simula que se enseña y que se aprende). 


\section{Referências}

AGUIRRE, J. M.; HAGGERTY, S. M.; LINDER, C. J. Student-teachers conceptions of science, teaching and learning: a case study in preservice science education. International Journal of Science Education, v. 12, n. 4 , p. 381-390, 1990.

; __ Preservice teachers meanings of learning. International Journal of Science Education, v. 17, n. 1, p. 119-131, 1995.

AMBROSE, R. Initiating change in prospective elementary school teachers' orientations to Mathematics teaching by building on beliefs. Journal of Mathematics Teacher Education, n. 7, p. 91-119, 2004.

APPLETON, K. Science activities that work: perceptions of primary school teachers. Research in Science Education, v. 32, p. 393-410, 2002.

AZCÁRATE, P. El conocimiento profesional: naturaleza, fuentes, organización y desarrollo. Quadrante, v. 8, p. 111-138, 1999a.

. Metodología de enseñanza. Cuadernos de Pedagogía, v. 276, p. 72-78, 1999b.

BESWICK, K. Changes in preservice teachers'attitudes and beliefs: the net impact of two mathematics education units and intervening experiences. School Science and Mathematics, v. 106, n. 1, p. 36-47, 2006.

BRYAN, L. A.; ABELL, S. K. Developmente of professional knowledge in learning to teaching elementary science. Journal of Research in Science Teaching, v. 36, n. 2, p. 121-139, 1999.

CAÑAL, P. Las actividades de enseñanza. Un esquema de clasificación. Investigación en la Escuela, v. 40, p. 5-21, 2000. 
CARDEÑOSO, J. M.; AZCÁRATE, P. Una estrategia de formación de maestros de matemáticas, basada en los ámbitos de investigación profesional (AIP). In: CONTRERAS, L. C.; BLANCO, L. Aportaciones a la formación inicial de maestros en el área de Matemáticas: una mirada a la práctica docente. Cáceres: Editorial Universidad de Extremadura, 2002. p. 186-226.

CHENG, M et. al. Pre-service teacher education students' epistemological beliefs and their conceptions of teaching. Teaching and Teacher Education, v. 25, n. 2, p. 319-327, 2009.

COBERN, W. Worldview theory and conceptual change in science education. Science Education, v. 80, n. 5, p. 579-610, 1996.

DUIT, R.; TREAGUST, D. Conceptual change: a powerful framework for improving science teaching and learning. International Journal of Science Education, v. 25, n. 6, p. 671-688, 2003.

FLORES, F. et al. Transforming science and learning concepts of physics teachers. International Journal of Science Education, v. 22, n. 2, p. 197-208, 2000.

FURIÓ, C. Tendencias actuales en la formación del profesorado de ciencias. Enseñanza de las Ciencias, v. 12, n. 2, p. 188-199, 1994.

GARCÍA, J. E.; García, F. F. Aprender investigando. Sevilla: Diada, 1989.

GUSTAFSON, B.J.; ROWELL, P. M. Elementary preservice teachers: constructing conceptions about learning science, teaching science and the nature of science. International Journal of Science Education, v. 17, p. 589-605, 1995.

HAEFNER, L. A.; ZEMBAL-SAUL, C. Learning by doing? Prospective elementary teachers' developing understandings of scientific inquiry 
and science teaching and learning. International Journal of Science Education, v. 26, n. 13, p. 1.653-1.674, 2004.

HANEY, J.; Mcarthur, J. Four case studies of prospective teachers' beliefs concerning constructivist practice. Science Education, v. 86, n. 6, p. 783-802, 2002.

HARRES, J. B. S. et al. Laboratórios de Ensino: inovação curricular na formação de professores de ciências. São Paulo: ESETec Editores Associados, 2005.

HASWE, M. Z. Effetcs of science teachers' epistemological beliefs in teaching. Journal of Research in Science Teaching, v. 33, n. 1, p. 47-63, 1996.

HEWSON, P. W.; HEWSON, M. G. Science teachers' concepctions of teaching: Implications for teacher education. International Journal of Science Education, v. 9, n. 4, p. 425-440, 1987.

JONES, M.; CARTER, G.; RUA, M. Children's concepts: tools for tranforming science teachers' knowledge. Science Education, v. 83, n. 5, p. 545-557, 1999.

JORAM, E.; GABRIELE, A. Preservice taechers' prior beliefs: transforming obstacles into opportunities. Teaching and Teacher Education, v. 12, n. 4, p. 365-384, 1988.

LAURIALA, A. Reformative in-service education for teachers (rinset) as collaborative action and learning enterprise: experiences from a finnish context. Teaching and Teacher Education, v. 14, n. 1, p. 53-66, 1998.

LEMBERGER, J.; HEWSON, P.; PARK, H. Relationships between prospective secondary teachers' classroom practice and their 
conceptions of biology and of teaching science. Science Education, v. 83, p. 347-371, 1999.

MARTÍN DEL POZO, R.; PORLÁN, R. (2001). Spanish prospective teachers' initial ideas about teaching chemical change. Chemistry Education Research and Practice in Europe, v. 2, p. 265-283, 2001.

; RIVERO, A. Construyendo un conocimiento profesionalizado para enseñar ciencias en la Educación Secundaria: los ámbitos de investigación profesional en la formación inicial del profesorado. Revista Interuniversitaria de Formación del Profesorado, v. 40, p. 63-79, 2001.

MELLADO, V. Concepciones y prácticas de aula de profesores de ciencias, en formación inicial de primaria y secundaria. Enseñanza de las Ciencias, v. 14, n. 3, p. 289-302, 1996.

MEYER, H. et al. Relationships between prospective elementary teachers' classroom practice and their conceptions of Biology and of Teaching Science. Science Education, v. 83, p. 323-346, 1999.

MORRISON, J. A.; LEDERMAN, N. G. Science teachers' diagnosis and understanding of students' preconceptions. Science Education, v. 87, n. 6, p. 849-867, 2003.

NIEDDERER, H.; GOLDBERG, F.; DUIT, R. Towards learning process studies: a review of the workshop on research in physics learning. In: NIEDDERER, H.; GOLDBERG, F; DUIT, R. (Ed.). Research in physics learning: theoretical issues and empirical studies. Proceedings of an international workshop. Germany: IPN, University of Kiel, 1992.

OSBORNE, J.; COLLINS, S. Pupils' views of the role and value of the science curriculum: a focus-group study. International Journal of Science Education, v. 23, n. 5, p. 441-467, 2001. 
PETERSON, R.; TREAGUST, D. Learning to teach Primary Science through problem-based learning. Science Education, v. 82, p. 215-237, 1998.

PIZZATO, M. C.; HARRES, J. B. S. Aprendizagem significativa e transformação na convivência: aproximações e indicadores para a formação de professores de ciências. Boletín de Estudios e Investigación Indivisa, v. 8, p. 429-439, 2007.

PORLÁN, R. Constructivismo y escuela. Sevilla: Diada, 1993.

; GARCÍA, S. The change of teachers' conceptions: a strategy forin-service science teachers' education. Teaching and Teacher Education, v. 8, n. 5/6, p. 537-548, 1992.

; MARTÍN DEL POZO, R. Ciencia, Profesores y Enseñanza: unas relaciones complejas. Alambique, v. 8, p. 23-32, 1996.

; __ Spanish teachers' epistemological and scientific conceptions: implications for teacher education. European Journal of Teacher Education, v. 25, n. 2/3, p. 151-169, 2002.

; . The conceptions of in-service and prospective primary school teachers about the teaching and learning of science. Journal of Science Teacher Education, v. 15, p. 39-62, 2004.

; - ¿Cómo progresa el profesorado al investigar problemas prácticos relacionados con la enseñanza de la ciencia? Alambique, v. 48, p. 92-99, 2006.

; RIVERO, A. El conocimiento de los profesores. Sevilla: Díada, 1998.

; ; MARTÍN DEL POZO, R. Conocimiento profesional y epistemología de los profesores I: Teoría, métodos e instrumentos. 
Enseñanza de las Ciencias, v. 15, n. 2, p. 155-171, 1997.

; . Conocimiento profesional y epistemología de los profesores II: Estudios empíricos y conclusiones. Enseñanza de las Ciencias, v. 16, n. 2, p. 271-288, 1998.

et al. (1996). Conocimiento profesional deseable y profesores innovadores: Fundamentos y principios formativos. Investigación en la Escuela, v. 29, p. 23-38, 1996.

et. al. Les obstacles à la formation professionnelle des professeurs en rapport avec leurs idées sur la science, l'enseignement et l'apprentissage. Aster, v. 26, p. 207-235, 1998.

RUSSELL, T.; MARTIN, A. Learning o teach science. In: ABELL, S.; LEDERMAN, N. (Ed.). Handbook of Reserch on science education. New Jersey: Lawrence Erlbaum Associates, 2007. p. 1.151-1.178.

SKAMP, K.; MUELLER, A. Student teachers' conceptions about effective primary science teaching: a longitudinal study. International Journal of Science Education, v. 23, n. 4, p. 331-351, 2001a.

;

A longitudinal study of the influences of primary and secondary school, university and practicum on student teachers' images of effective primary science practice. International Journal of Science Education, v. 23, n. 3, p. 227-245, 2001 b.

SO, W.; WATKINS, D. A. From beginning teacher education to professional teaching: a study of the thinking of Hong Kong primary science Teachers. Teaching and Teacher Education, v. 21, n. 5, p. 525541, 2005.

SZYDLIK, J. E.; SZYDLIK, S. D.; BENSON, S. R. Exploring changes in Pre-Service Elementary Teachers'mathematical beliefs. Journal of 
Mathematics Teacher Education, v. 6, p. 253-279, 2003.

TILLEMA, H. H. Belief change towards self-directed learning in student teachers: immersion in practice or reflection on action. Teaching and Teacher Education, v. 16, p. 575-591, 2000.

; VAN DER WESTHUIZEN, G. J. Knowledge construction in collaborative enquiry among teachers. Teachers and Teaching: theory and practice, v. 12, n. 1, p. 51-67, 2006.

WATTERS, J.; GINNS, I. Developing motivation to teach elementary science: effect of collaborative and authentic learning practices in preservice education. Journal of Science Teacher Education, v. 11, n. 4, p. 301-321, 2000.

WATTS, M.; JOFILI, Z. Towards critical constructivist teaching. International Journal of Science Education, v. 20, n. 2, p. 173-185, 1998.

YERRICK, R.; PARKER, H.; NUGENT, J. Struggling to promote deeply rooted change: the "filtering efect" of teachers' beliefs on understanding transformational views of teaching science. Science Education, v. 81, n. 2, p. 137-159, 1997.

ZELLERMAYER, M.; TABAK, E. Knowledge construction in a teachers' community of enquiry: a possible road map. Teachers and Teaching: theory and practice, v. 12, n. 1, p. 33-49, 2006.

ZEMBAL-SAUL, C.; BLUMENFELD, P.; KRAJCIK, J. (2000). Influence of guided cycles of planning, teaching, and reflection on Prospective Elementary Teachers' Science content representations. Journal of Research in Science Teaching, v. 37, n. 4, p. 318-339, 2000. 


\title{
The progression of conceptions of prospective teachers primary on teaching methodology
}

\begin{abstract}
This article describes the evolution of prospective primary teachers' conceptions of the methodology of teaching. Three categories were analyzed: the concept of activity, the organization of activities, and the concept of teaching resources, but in this paper only show the organization of activities. The study was conducted with five teams of prospective teachers, who were participating in teacher education courses of a constructivist orientation. The results showed very different itineraries in the processes of change, and the presence of two major obstacles - the belief that teaching is the direct cause of learning, and epistemological absolutism. The study allows us to deduce some implications for initial teacher education.

Keywords: pre-service teacher education; professional knowledge; methodological strategies; organization activities.
\end{abstract}

